\title{
OPERATORS WITH MINIMAL PSEUDOSPECTRA AND CONNECTIONS TO NORMALITY
}

\author{
SAMIR RAOUAFI
}

Abstract. This paper mainly studies the class of bounded linear operators $A$ with minimal pseudospectra $\sigma_{\varepsilon}(A)=\sigma(A)+\mathbb{D}_{\varepsilon}$ for some $\varepsilon>0$, where $\sigma(A)$ denotes the spectrum of $A$, and $\mathbb{D}_{\varepsilon}$ denotes the open disk of radius $\varepsilon$ centered at the origin. Some characterizations of the normality of operators with minimal pseudospectra are provided in terms of only one $\varepsilon$-pseudospectrum. Furthermore, a characterization of the normality of arbitrary $N \times N$ complex matrices is given for $N \leqslant 4$. Some applications to numerical ranges are also presented.

Mathematics subject classification (2010): 47A10, 47A12, 47A20, 47B15.

Keywords and phrases: Pseudospectra, numerical range, normal operator, dilation.

\section{REFERENCES}

[1] Y. A. Abramovich and C. D. Aliprantis, An Invitation to Operator Theory, American Mathematical Society, Providence, RI, 2002.

[2] S.K. Berberian, Some Conditions of an Operator Implying Normality. II, Proc. Amer. Math. Soc., 26, 2, 1970, 277-281.

[3] S.K. Berberian, Some Conditions of an Operator Implying Normality. III, Proc. Japan Acad., 46, 7 , 1970, 630-632.

[4] S.K. BERBERIAN, An extension of Weyl's theorem to a class of not necessarily normal operators, Michigan Math. Jour, 16, 3, 1969, 273-279.

[5] S.K. Berberian, Approximate proper vectors, Proc. Amer. Math. Soc., 13, 1, 1962, 111-114.

[6] A. Böttcher And S. M. Grudsky, Spectral Properties of Banded Toeplitz Matrices, SIAM, Philadelphia, 2005.

[7] A. BötTCher And B. Silberman, Introduction to Large Truncated Toeplitz Matrices, SpringerVerlag, New York, 1999.

[8] M. CROUZEIX AND C. PALEnCIA, The numerical range is a $1+\sqrt{2}$-spectral set, SIAM J. Matrix Anal. Appl., 38, 2, 2017, 649-655.

[9] M. CRouzeix, A functional calculus based on the numerical range: applications, Linear and Multilinear Algebra, 56, 1-2, 2008, 81-103.

[10] J. CUI, C. K. LI, AND Y. T. Poon, Pseudospectra of special operators and pseudospectrum preservers, J. Math. Anal. Appl., 419, 2, 2014, 1261-1273.

[11] L. Elsner And KH. D. IKramov, Normal Matrices: An Update, Linear Algebra Appl., 285, 1-3, 1998, 291-303.

[12] M. R. EMBry, Conditions implying normality in Hilbert space, Pacific J. Math., 18, 3, 1966, 457-460.

[13] R. Grone, C. R. Johnson, E. M. SA, And H. Wolkowicz, Normal Matrices, Linear Algebra Appl., 87, 1, 1987, 213-225.

[14] B. Jia And Y. Feng, On pseudospectral radii of operators on Hilbert spaces, Ann. Funct. Anal., 9 , 4, 2018, 474-484.

[15] C. R. Johnson, Normality and the numerical range, Linear Algebra Appl., 15, 1, 1976, 89-94.

[16] V. Paulsen, Completely Bounded Maps and Operator Algebras, Cambridge Studies in Advanced Mathematics 78, Cambridge University Press, 2003.

[17] T. Ransford And S. RAOUAFI, Pseudospectra and holomorphic functions of matrices, Bull. London Math. Soc., 45, 4, 2013, 693-699. 
[18] S. RaouAfi, A generalization of the Kreiss Matrix Theorem, Linear Algebra Appl., 549, 1, 2018, 86-99.

[19] L. Reichel AND L. N. Trefethen, Eigenvalues and Pseudo-eigenvalues of Toeplitz Matrices, Linear Algebra Appl., 162-164, 1, 1992, 153-185.

[20] J. G. StAmpfli, Normality and the numerical range of an operator, Bull. Amer. Math. Soc., 72, 6, 1966, 1021-1022.

[21] J. G. Stampfli And J. P. Williams, Growth conditions and the numerical range in a Banach algebra, Tohoku Math. Journ., 20, 4, 1968, 417-424.

[22] L.N. TREFETHEN AND M. EMBREE, Spectra and Psedospectra, The behavior of nonnormal matrices and Operators, Princeton Unviersity Press, Princeton, 2005.

[23] J. P. Williams, Similarity and the Numerical Range, J. Math. Anal. Appl., 26, 2, 1969, 307-314. 